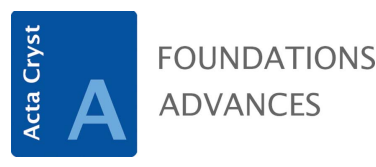

ISSN 2053-2733

Received 19 October 2018

Accepted 28 August 2019

Edited by L. D. Marks, Northwestern University, USA

Keywords: interference fringes; mirage fringes; X-ray beam trajectory; bent crystal; multiple Bragg diffraction; dynamical theory of X-ray diffraction.

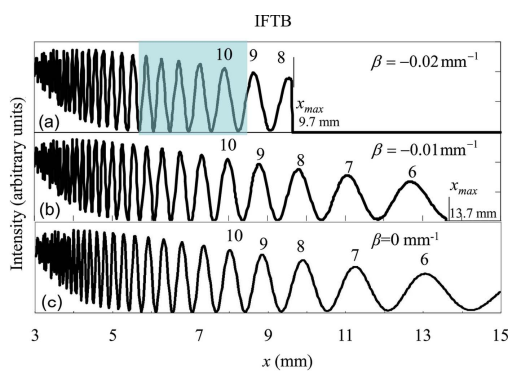

OPEN ๑ ACCESS

\section{X-ray interference fringes from a weakly bent plane- parallel crystal with negative strain gradient}

\author{
Tomoe Fukamachi, ${ }^{\mathrm{a} *}$ Sukswat Jongsukswat, ${ }^{\mathrm{b}}$ Dongying Ju, ${ }^{\mathrm{a}}$ Riichirou Negishi, ${ }^{a}$ \\ Keiichi Hirano $^{\mathrm{c}}$ and Takaaki Kawamura ${ }^{\mathrm{d}}$
}

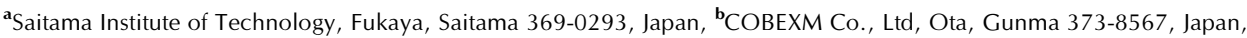
${ }^{\mathbf{c}}$ Institute of Material Structure Science, KEK-PF, High Energy Accelerator Research Organization, Tsukuba, Ibaraki 3050801, Japan, and d Center for Ceramic Matrix Composites, Tokyo University of Technology, Hachioji, Tokyo 192-0982, Japan. *Correspondence e-mail: tomoe-f@wonder.ocn.ne.jp
\end{abstract}

Under the anomalous transmission condition in the Bragg mode, X-ray interference fringes were observed between two beams with different hyperbolic trajectories in a very weakly bent plane-parallel perfect crystal with negative strain gradient. The origin of the fringes was analysed based on the dynamical theory of diffraction for a distorted crystal. In the reflected beam from the entrance surface, the interference fringes were observed between once- and twice-reflected beams from the back surface. In the transmitted beam from the back surface, the interference fringes were observed between the direct beam and once-reflected beam from the entrance surface. In the emitted beam from the lateral surface, the interference fringes were observed between the beams after different numbers of reflections in the crystal. The multiply reflected beams were formed by a combined result of long propagation length along the beam direction with large divergence of the refracted beams when the strain gradient was negative. The period of these interference fringes was sensitive to very weak strain, of the order of $10^{-7}$.

\section{Introduction}

When X-rays are incident on a thin plane-parallel perfect crystal in the symmetric Bragg geometry, the refracted beam in the crystal reaches the back surface as illustrated in Fig. $1(a)$. A part of the beam is reflected $\left(\mathbf{S}_{4}\right)$ and the rest is emitted from the back surface as the transmitted beam $\left(P_{\mathrm{t}}\right)$. The refracted beam is referred to as the beam corresponding to the Poynting vector excited at a point on the dispersion surface defined in the dynamical theory of diffraction. The refracted beam is called the wavefield by Authier (2001). In the twowave approximation, it is composed of two waves: one is propagating in the forward direction and the other in the diffracted-wave direction. In an anomalous transmission condition, the divergence of the refracted beam is much larger than that of the incident wave (Authier, 2001). If the divergence angle of the refracted beam is large enough, interference fringes can be formed between once $\left(\mathbf{S}_{1}\right)$ - and twice $\left(\mathbf{S}_{2}\right)$-reflected beams from the back surface (abbreviated as IFRB) as shown in Fig. 1(a). Similar interference fringes can be observed in the transmitted beam (IFTB) from the back surface. If the crystal is small along the incident azimuth direction $(x)$ compared with the propagation length of the $\mathrm{X}$-ray, interference fringes can be observed in the emitted beams from the lateral surface both in the diffracted- (IFLSD) and the transmitted-wave directions (IFLST). Both IFLSD and IFLST have been observed by Fukamachi et al. (2004, 2005) from a thin Ge plane-parallel crystal. These fringes were 
formed by interference between the beams directly propagating to the lateral surface $\left(\mathbf{S}_{3}\right)$ and once reflected from the back surface $\left(\mathbf{S}_{4}\right)$ (Hirano et al., 2008, 2009a,b; Fukamachi, Hirano et al., 2011).

When a perfect crystal is bent with positive strain gradient (strain gradient parameter defined below $\beta>0$ ), the trajectory of the refracted beam shows a hyperbolic form opening up and
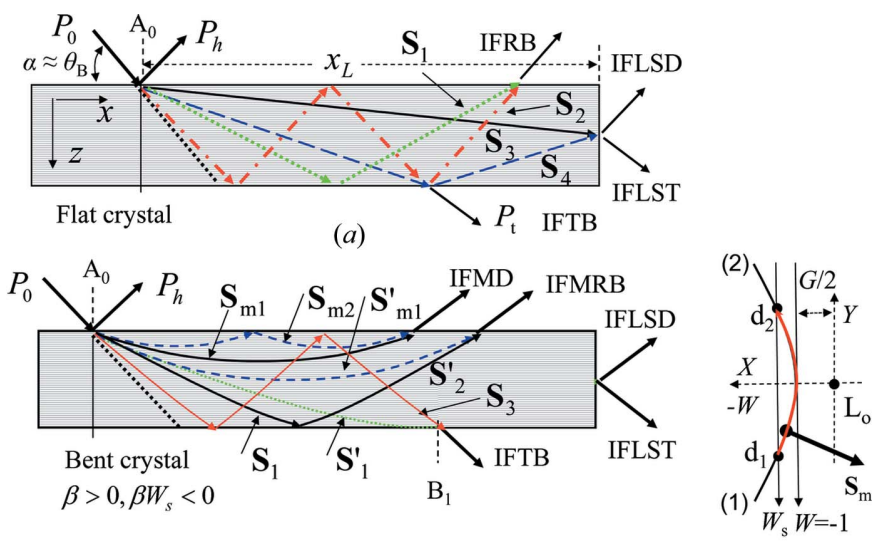

Figure 1

(b)

(c)

Schematic illustrations of the beam trajectories in a plane-parallel crystal. (a) Beam trajectories in an unbent crystal. $P_{0}$ represents the incident wave on the surface, $P_{\mathrm{h}}$ the diffracted wave and $P_{\mathrm{t}}$ the transmitted wave. (b) Beam trajectories in a bent crystal when the X-ray is incident on the expanded surface due to bending $(\beta>0)$. $\mathbf{S}_{1}^{\prime}$ represents the beam touching the back surface at $\mathbf{B}_{1}$ and $\mathbf{S}_{3}$ the beam reflected from the entrance surface after reflection from the back surface. $(c)$ The variation range of the tie point on the dispersion surface for mirage beams. $d_{1}$ on branch (1) is the tie point at the incident point and $d_{2}$ on branch (2) is that at the emitted point. $\mathrm{L}_{\mathrm{o}}$ is the Lorentz point and $G$ the Bragg gap. The distance from the incident point of the X-ray $\left(\mathrm{A}_{0}\right)$ to the edge of the crystal is $x_{L}$.

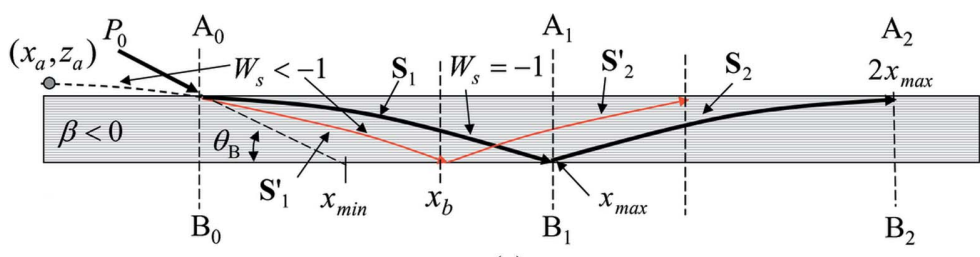

(a)

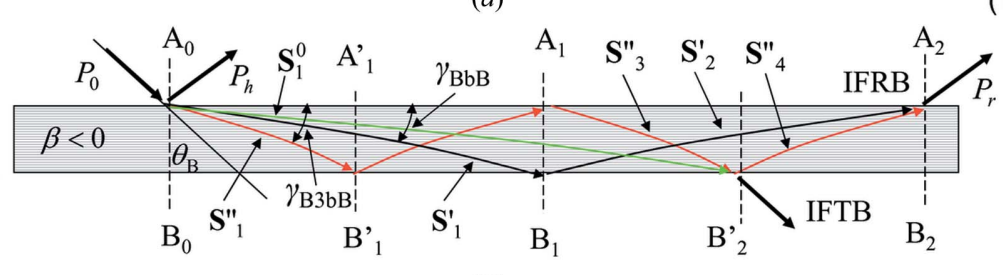

(b)

Figure 2

Schematic illustrations of the beam trajectories in a bent plane-parallel crystal for $\beta<0$. (a) $\mathbf{S}_{1}$ represents the beam excited when $W_{\mathrm{s}}=-1$. It propagates the longest distance $\left(x_{\max }\right)$ in the $x$ direction, reaches the back surface at $B_{1}$ and is partly reflected as the beam $\mathbf{S}_{2} . \mathbf{S}^{\prime}{ }_{1}$ represents a beam excited when $W_{\mathrm{s}}<-1$ and $\mathbf{S}_{2}^{\prime}$ the corresponding reflected beam from the back surface. $(b)$ Beam trajectories to form IFRB and IFTB. IFRB from $\mathrm{A}_{2}$ are formed by interference between the beam $\mathbf{S}_{2}{ }_{2}$ once reflected and the beam $\mathbf{S}^{\prime \prime}{ }_{4}$ twice reflected from the back surface. IFTB from $\mathrm{B}_{2}^{\prime}$ are formed by interference between the beam $\mathbf{S}_{1}^{0}$ reaching directly the back surface and the beam $\mathbf{S}_{3}^{\prime \prime}$ once reflected from the back surface. (c) The variation range of the tie point on the dispersion surface for $\mathbf{S}_{1}^{\prime}$ and $\mathbf{S}_{2}^{\prime}$. The range from $d_{1}$ to $d_{2}$ on branch (1) is for $\mathbf{S}_{1}^{\prime}$ and that from $d_{2}^{\prime}$ to $d^{\prime}{ }_{1}$ on branch (2) is for $\mathbf{S}_{2}^{\prime} \cdot \gamma_{\mathrm{BbB}}$ and $\gamma_{\mathrm{B} 3 \mathrm{bB}}$ are the angles of the refracted beams in the $\mathrm{BbB}$ and the $\mathrm{B} 3 \mathrm{bB}$ modes from the surface, respectively.

some refracted beams return to the entrance surface without reaching the back surface such as $\mathbf{S}_{\mathrm{m} 1}, \mathbf{S}_{\mathrm{m} 2}$ and $\mathbf{S}_{\mathrm{m} 1}^{\prime}$ in Fig. 1(b), as pointed out by Gronkowski \& Malgrange (1984). These refracted beams are called mirage beams by Authier (2001). Fig. 1(c) shows the tie points corresponding to mirage beams in the range from $d_{1}$ to $d_{2}$ on the dispersion surface. The interference fringes formed by two mirage diffraction beams (IFMD) have been observed by Fukamachi et al. (2010) and Jongsukswat et al. (2012a, 2013). It was pointed out by Fukamachi, Jongsukswat et al. (2011) and Jongsukswat et al. $(2012 b)$ that interference fringes between the mirage diffrac$\mathbf{S}^{\prime}{ }_{2}$ (IFMRB) are expected to be observed as shown in Fig. 1(b).

If a perfect crystal is bent with negative strain gradient $(\beta<0)$, the trajectory of the refracted beam shows a hyperbolic form opening down and the beam always reaches the back surface as shown in Fig. 2(a). Fig. 2(c) shows that the tie points corresponding to the refracted beam $\mathbf{S}_{1}^{\prime}$ and $\mathbf{S}_{2}^{\prime}$ move reflected twice from the back surface in Fig $2(b), \gamma$ is the angle of the refracted beam from the surface at the incident point. Similar interference fringes can be observed between two beams $n$ and $n+1$ ( $n$ is a positive integer) times reflected from the back surface. IFTB also can be observed for $\beta<0$.

In this paper, we will report on the measurement of IFRB, IFTB, IFLSD and IFLST from a weakly bent Si plane-parallel fringes with those for $\beta>0$. We will analyse the interference

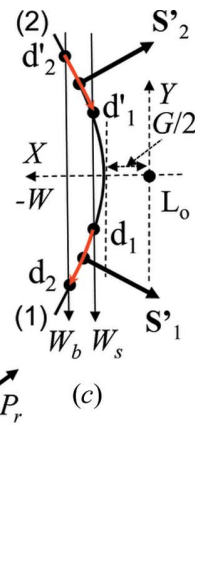
diffraction for a distorted crystal and reveal some characteristics of them for $\beta<0$.

\section{Experimental}

The sample was a plane-parallel single $\mathrm{Si}$ crystal. The top (entrance) and bottom (back) surfaces of the crystal were polished by a non-disturbance polishing method at Sharan Inc. The size was $50 \mathrm{~mm}$ long, $15 \mathrm{~mm}$ wide and $0.28 \mathrm{~mm}$ thick. One end of the sample was clamped and the other end was free along the gravity direction as shown in Fig. 3(b). The sample was bent due to gravity and the residual strain. The experiments were carried out using $\mathrm{X}$-rays from synchrotron radiation at the bending-magnet beamline 15C, Photon Factory, Tsukuba, Japan. The measuring optical system is shown in Fig. 3(a). The X-rays were $\sigma$-polarized and had a very narrow band of energy achieved by using an $\mathrm{Si}(111)$ double- 

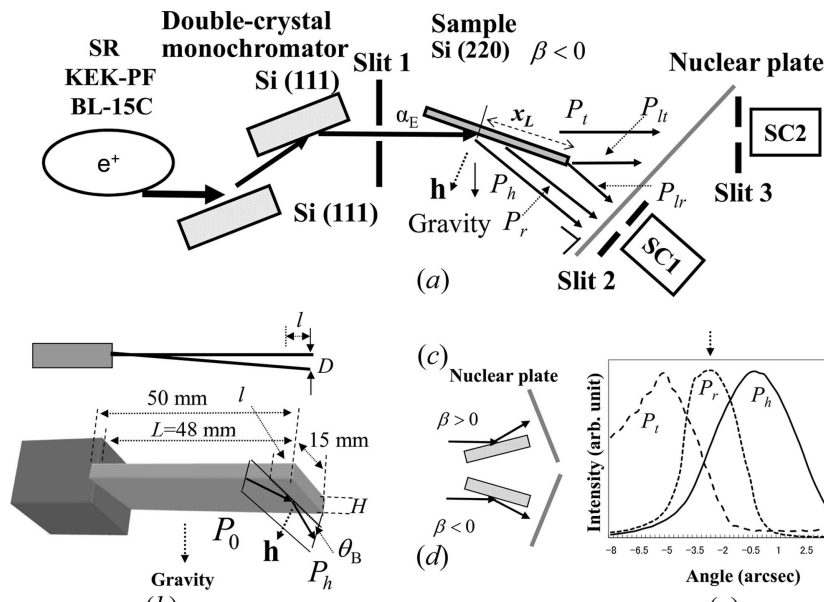

(b)

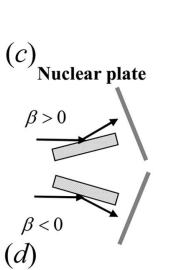

(d)

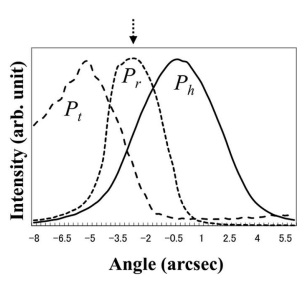

(e)

Figure 3

(a) A schematic diagram of the measuring system. $x_{L}$ is the distance between the incident point of the X-rays and the crystal edge of the entrance surface. SC1 and SC2 are the scintillation counters set up in the reflected- and transmitted-wave directions, respectively. (b) Sample geometries. $(c)$ and $(d)$ the incident- and reflected-wave geometries with respect to the samples for $\beta>0$ and $\beta<0$, respectively. (e) The measured rocking curves of $P_{\mathrm{h}}, P_{\mathrm{t}}$ and $P_{\mathrm{r}}$.

crystal monochromator. The X-ray energy was $11100 \mathrm{eV}$, which was determined by measuring XANES (X-ray absorption near-edge structure) from a thin Ge plate near the Ge $K$ absorption edge $(11103 \mathrm{eV})$ with an accuracy of $\pm 0.5 \mathrm{eV}$. The distance from the source to slit 1 was $30 \mathrm{~m}$ and that from slit 1 to the sample was $300 \mathrm{~mm}$. The vertical width of slit 1 was $0.02 \mathrm{~mm}$. In Fig. 3( $a), P_{\mathrm{h}}, P_{\mathrm{r}}, P_{\mathrm{t}}, P_{\mathrm{lr}}$ and $P_{\mathrm{lt}}$ are the intensities of the diffracted wave, the reflected beam except for the diffracted beam, the transmitted beam, the emitted beam from the lateral surface in the diffracted-wave direction and that in the transmitted-wave direction, respectively. As shown in Fig. $3(b)$, the X-rays were incident on the crystal with the azimuth

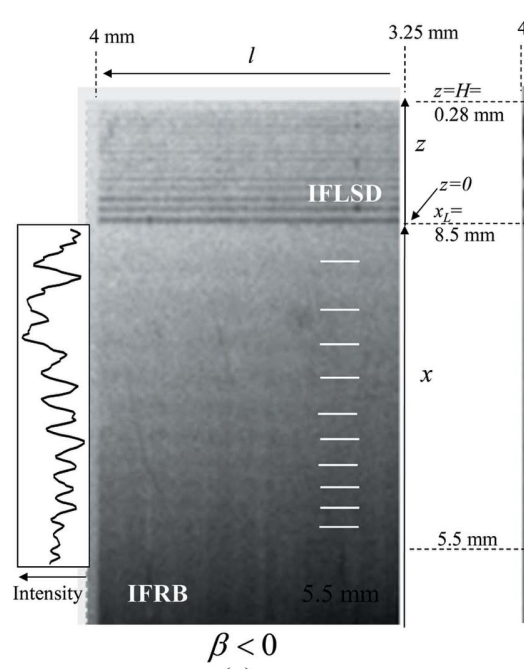

(a)

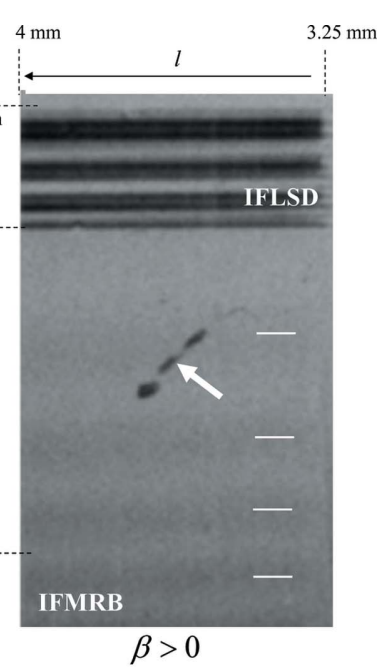

(b)

Figure 4

Section topographs of $\mathrm{Si}(220)$ in the Bragg mode recorded in the diffracted-wave direction for $(a) \beta<0$ and (b) $\beta>0$. The distance $x_{L}$ is $8.5 \mathrm{~mm}$. The left side of $(a)$ shows the digitized intensities of IFRB. The horizontal direction is the distance $l$.

perpendicular to the bending direction. The incident glancing angle was fixed at the angle where the rocking curve of $P_{\mathrm{r}}$ in Fig. 3(e) showed the peak and the anomalous transmission was maximized. The X-ray intensities were measured by the scintillation counters (SC1 and SC2) and recorded on a nuclear plate (ILFORD L4; emulsion thickness, $25 \mu \mathrm{m}$ ). The incident and reflected X-ray geometries with respect to the sample are shown for positive and negative values of $\beta$ in Figs. $3(c)$ and $3(d)$, respectively. For observation of IFRB, it is important to make the value of $|\beta|$ small. If $l$ denotes the distance from the free edge to the incident plane and $L$ that from the free edge to the fixed edge, $|\beta|$ is proportional to $l^{2} / L^{4}$ (Jongsukswat et al., 2013). In the present experiment the distance $l$ was between 3.25 and $4 \mathrm{~mm}$, and $L$ was $48 \mathrm{~mm}$.

Fig. 4 shows the section topographs of $\mathrm{Si}(220)$ measured in the diffracted direction under only the gravity force. Fig. 5 shows those measured in the transmitted-wave direction. The strain gradient was negative in Fig. 4(a) and Fig. 5(a), and positive in Fig. 4(b) and Fig. 5(b). The exposure time for taking one topograph was $120 \mathrm{~min}$ for Fig. 4(a), $60 \mathrm{~min}$ for Fig. 5(a), $15 \mathrm{~min}$ for Fig. 4(b) and $30 \mathrm{~min}$ for Fig. 5(b). In Fig. 4(a) the interference fringes in the upper part are attributed to IFLSD and those in the lower part to IFRB. The vertical direction of the lower part corresponds to the distance $x$ from the incident point of the X-rays on the entrance surface and that in the upper part corresponds to the distance $z$ from the edge of the entrance surface on the lateral surface. The horizontal direction corresponds to distance $l$. Ten dark lines are observed in IFRB as indicated by white lines. The plot of digitized intensities of IFRB is given on the left side of the figure, showing ten peaks in the range of $x$ between 5.5 and $8.5 \mathrm{~mm}$. The number of interference fringes in Fig. 4(a) is larger than that in Fig. 4(b) where the number is three in the same range of $x$. The distance between two adjacent dark lines in IFRB increases as a function of $x$, which is a similar

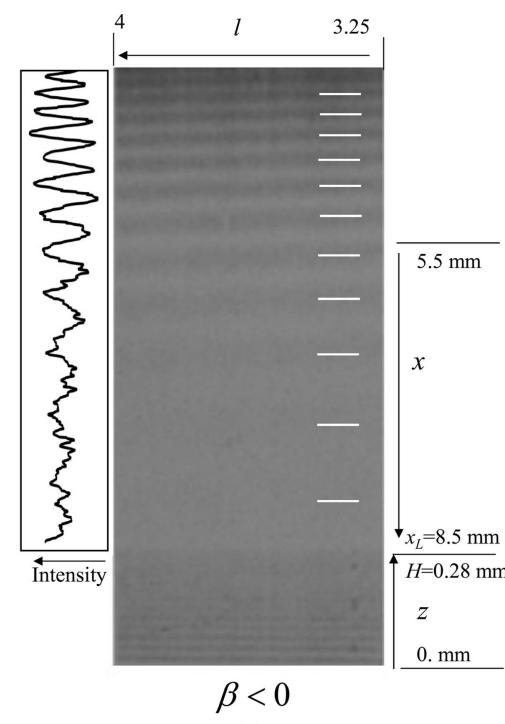

(a)

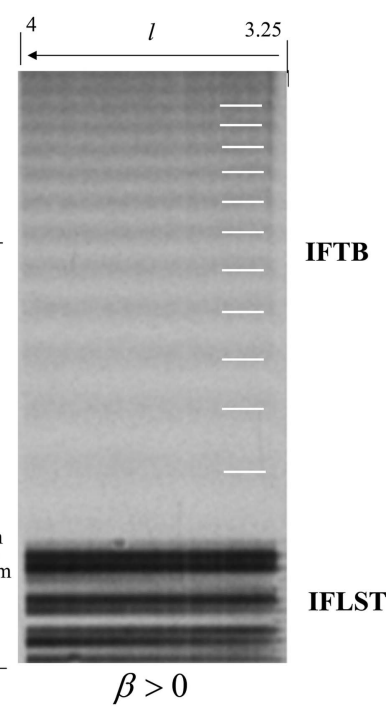

(b)
Figure 5

Section topographs of $\mathrm{Si}(220)$ in the Bragg mode recorded in the transmitted-wave direction for $(a) \beta<0$ and $(b) \beta>0$. The distance $x_{L}$ is $8.5 \mathrm{~mm}$. The left side of $(a)$ shows the digitized intensities of IFTB. 
variation to that in IFMRB shown in Fig. 4(b). The distance between two adjacent dark lines in IFLSD in Fig. 4(a) is smaller than that in Fig. 4(b). The dark contrast indicated by the white arrow in Fig. 4(b) is accretions on the nuclear emulsion plate. In Fig. 5(a) the interference fringes in the upper part are attributed to IFTB and those in the lower part to IFLST. The number of dark lines in IFTB indicated by white lines is five in the same range of $x$. The horizontal direction is the same as in Fig. 4. The distance between two adjacent dark lines increases as a function of $x$, which is a similar variation to that in IFRB. In Fig. 5(b) the interference fringes in the upper part are attributed to IFTB and those in the lower part to IFLST. The number of dark lines in ITFB for $\beta<0$ is almost the same as that for $\beta>0$ in the same range of $x$.

\section{Theoretical basis}

\subsection{Beam trajectory}

In the dynamical theory of diffraction, the deviation parameter $W$ from an exact Bragg condition is defined by

$$
W=\frac{\sin 2 \theta_{\mathrm{B}}}{C\left(\chi_{h} \chi_{-h}\right)^{1 / 2}}\left[\alpha-\left(\theta_{\mathrm{B}}+\frac{\left|\chi_{0}\right|}{\sin 2 \theta_{\mathrm{B}}}\right)\right] .
$$

Here $\alpha$ is the incident glancing angle, $\theta_{\mathrm{B}}$ is the Bragg angle, $C$ the polarization factor and $\chi_{h}$ the hth Fourier component of $\mathrm{X}$-ray polarizability. By using the deviation parameter at the incident point $\left(W_{\mathrm{s}}\right)$, the trajectory of the refracted beam for $\left|W_{\mathrm{s}}\right| \geq 1$ in a non-absorbing bent crystal with constant strain gradient is given by

$$
\left(\frac{\beta z}{\tan \theta_{\mathrm{B}}}+W_{\mathrm{s}}\right)^{2}-\left[\beta x+s\left(W_{\mathrm{s}}\right)\left(W_{\mathrm{s}}^{2}-1\right)^{1 / 2}\right]^{2}=1,
$$

according to Gronkowski \& Malgrange (1984). The coordinates $x$ and $z$ are parallel and inward normal to the crystal surface and the origin is taken at the incident point of the $\mathrm{X}$-ray. $s\left(W_{\mathrm{s}}\right)$ is equal to 1 for $W_{\mathrm{s}}>1$ and -1 for $W_{\mathrm{s}}<-1$. The strain gradient parameter $\beta$ is given by

$$
\beta=\frac{\lambda}{C\left(\chi_{h} \chi_{-h}\right)^{1 / 2}} \frac{\partial^{2}(\mathbf{h} \cdot \mathbf{u})}{\partial x_{0} \partial x_{h}},
$$

where $\mathbf{h}$ is the reciprocal-lattice vector, $\mathbf{u}$ the displacement vector of an atom and $\lambda$ the $\mathrm{X}$-ray wavelength. $x_{0}$ and $x_{h}$ are the coordinates of the transmitted- and the diffracted-wave directions, respectively. According to Yan \& Noyan (2006) and Yan et al. (2007), $\beta$ is related to the strain gradient $\varepsilon_{z z}^{\prime}$ as

$$
\beta=\frac{2 \sin ^{2} \theta_{\mathrm{B}} \tan \theta_{\mathrm{B}}}{C\left(\chi_{h} \chi_{-h}\right)^{1 / 2}} \varepsilon_{z z}^{\prime} .
$$

The strain in a bent plane-parallel crystal of thickness $H$ is given by

$$
\varepsilon_{z z}=\frac{d-d_{0}}{d_{0}}=\varepsilon_{z z}^{\prime}\left(z-\frac{H}{2}\right),
$$

where $d$ and $d_{0}$ are the distances between two adjacent lattice planes normal to the crystal surface with and without distortion due to bending, respectively.
The trajectory of the refracted beam becomes a hyperbola for $\beta \neq 0$ by using equation (2). Its vertex position $\left(x_{a}, z_{a}\right)$ is given by

$$
\begin{gathered}
x_{a}=\frac{-s\left(W_{\mathrm{s}}\right)\left(W_{\mathrm{s}}^{2}-1\right)^{1 / 2}}{\beta}, \\
z_{a}=\frac{-\tan \theta_{\mathrm{B}}}{\beta}\left[W_{\mathrm{s}}-s\left(W_{\mathrm{s}}\right)\right] .
\end{gathered}
$$

The beam trajectory for positive strain gradient has been studied in previous works (Fukamachi et al., 2010; Fukamachi, Jongsukswat et al., 2011; Jongsukswat et al., 2012a,b, 2013). The vertex of the hyperbola is located inside the crystal when $\beta>0$ and $W_{\mathrm{s}}<-1$ according to equation (7). Some refracted beams such as $\mathbf{S}_{\mathrm{m} 1}$ and $\mathbf{S}_{\mathrm{m} 2}$ return to the entrance surface without touching the back surface as depicted in Fig. 1(b). On the other hand, when the strain gradient is negative $(\beta<0)$, the vertex is located outside the entrance surface. The beam trajectory is bent downwards in the crystal and all the beams reach the back surface as depicted in Figs. $2(a)$ and $2(b)$. The refracted beam corresponding to $W_{\mathrm{s}}=-1$ propagates in the crystal when $\beta<0$, while such a refracted beam is not excited when $\beta>0$. Using equation (2), the value of the deviation parameter $W_{\mathrm{b}}$ at the back surface is given by

$$
W_{\mathrm{b}}=\frac{\beta H}{\tan \theta_{\mathrm{B}}}+W_{\mathrm{s}}
$$

which satisfies the relation $W_{\mathrm{b}}<W_{\mathrm{s}} \leq-1$. The $x$ component of the propagation length of the X-ray from the incident point to the back surface is given as

$$
x_{\mathrm{b}}=\frac{1}{|\beta|}\left\{\left[\left(\frac{|\beta| H}{\tan \theta_{\mathrm{B}}}+\left|W_{\mathrm{s}}\right|\right)^{2}-1\right]^{1 / 2}-\left(W_{\mathrm{s}}^{2}-1\right)^{1 / 2}\right\},
$$

by setting $z=H$ in equation (2). The distance $x_{\mathrm{b}}$ decreases monotonically as a function of $\left|W_{\mathrm{s}}\right|$ for $W_{\mathrm{s}} \leq-1$.

The maximum value of $x$ for observing IFTB is determined by the exit point of the refracted beam for $\gamma=0\left(W_{\mathrm{s}}=-1\right)$. The value $\left(x_{\max }\right)$ is half the maximum value of $x$ for observing IFRB ( $\left.2 x_{\max }\right)$ for $\gamma=0$ as depicted in Fig. 2(a). Using equation (9), the value of $x_{\max }$ is $9.6,7.9$ and $6.8 \mathrm{~mm}$ when $|\beta|$ is 0.02 , 0.03 and $0.04 \mathrm{~mm}^{-1}$, respectively. In the experiment, IFTB is observed up to $8 \mathrm{~mm}$ as shown in Fig. $5(a) .|\beta|$ must be less than $0.03 \mathrm{~mm}^{-1}$. The uppermost observed point $z_{0}$ of IFLSD is given by the exit point of the beam for $\gamma=0$ from the lateral surface. The point $z_{0}$ is $0.21,0.11$ and $0.055 \mathrm{~mm}$ when $|\beta|$ is $0.02,0.01$ and $0.005 \mathrm{~mm}^{-1}$, respectively. As the measured value of $z_{0}$ is around $0.14 \mathrm{~mm},|\beta|$ must be more than $0.01 \mathrm{~mm}^{-1}$. Then it is reasonable to take the value of $|\beta|$ as $0.02 \mathrm{~mm}^{-1}$ within the error of $\pm 0.01 \mathrm{~mm}^{-1}$. To determine the value of $\beta$ from only IFRB, it is necessary to measure the interference fringes for $x$ more than $17 \mathrm{~mm}$ as will be shown later. 
3.2. Interference fringes between two reflected beams from the back surface

In Fig. 2(b), as IFRB emitted from $\mathrm{A}_{2}$ are observed in the range of $x$ from around 5.5 to $8.5 \mathrm{~mm}$, they are attributed to the interference fringes between the once-reflected beam $\left(\mathbf{S}_{2}^{\prime}\right)$ and the twice-reflected beam $\left(\mathbf{S}^{\prime \prime}{ }_{4}\right)$ from the back surface. The beam once reflected from the back surface is called the beam in the $\mathrm{BbB}$ mode hereafter, since it is incident on the entrance surface $\left(A_{0}\right)$ satisfying the boundary condition of the Bragg mode, reflected from the back surface $\left(B_{1}\right)$ satisfying that of the Bragg mode and emitted from the entrance surface $\left(A_{2}\right)$ satisfying that of the Bragg mode. It is possible for these beams to interfere with the beam in the B5bB mode. But the amplitude of the beams in the B5bB mode is much smaller than that in the $\mathrm{BbB}$ mode, because the amplitude becomes small after each reflection from the entrance or the back surface. In the following, the contribution of the beam in the $\mathrm{B} 5 \mathrm{bB}$ mode is ignored.

The amplitude of the beam in the BbB mode is denoted as $E_{\mathrm{BbB}}$. Similarly, the amplitude corresponding to the beam twice reflected from the back surface, i.e. the beam in the $\mathrm{B} 3 \mathrm{bB}$ mode, is denoted as $E_{\mathrm{B} 3 \mathrm{bB}}$. Then the amplitudes of $E_{\mathrm{BbB}}$ and $E_{\mathrm{B} 3 \mathrm{bB}}$ from a non-absorbing crystal can be written as

$$
\begin{aligned}
& E_{\mathrm{BbB}}=R_{1} \exp \left(2 \pi i \theta_{1}\right) E_{0}, \\
& E_{\mathrm{B} 3 \mathrm{bB}}=R_{2} \exp \left(2 \pi i \theta_{2}\right) E_{0} .
\end{aligned}
$$

Here $E_{0}$ is the amplitude of the incident X-ray. $\theta_{1}$ and $\theta_{2}$ are the phase factors of $E_{\mathrm{BbB}}$ and $E_{\mathrm{B} 3 \mathrm{bB}}$, respectively. The phase factor $\theta_{m}(m=1$ and 2$)$ is given by

$$
\theta_{m}=\int \mathbf{k}_{m}\left(\mathbf{r}_{m}\right) \cdot d \mathbf{r}_{m},
$$

with $\mathbf{k}_{m}$ being the wavevector and $\mathbf{r}_{m}$ the position vector. $\theta_{m}$ can be divided into two parts as

$$
\theta_{m}=\theta_{x m}+\theta_{z m} \text {. }
$$

$\theta_{x m}$ and $\theta_{z m}$ are obtained from the $x$ and $z$ components of $\mathbf{r}_{m}$. The details of the calculation of equation (13) were given by Fukamachi et al. (2010) and Jongsukswat et al. (2012b). In equations (10) and (11), $R_{1}$ and $R_{2}$ are given by

$$
\begin{aligned}
& R_{1}=r_{1}\left(W_{\mathrm{s} 1}\right)\left[\frac{r_{1}\left(W_{\mathrm{s} 1}\right)}{r_{2}\left(W_{\mathrm{b} 1}\right)}-1\right], \\
& R_{2}=\frac{r_{1}^{2}\left(W_{\mathrm{s} 2}\right)}{r_{2}\left(W_{\mathrm{b} 2}\right)}\left[\frac{r_{1}\left(W_{\mathrm{s} 2}\right)}{r_{2}\left(W_{\mathrm{b} 2}\right)}-1\right] .
\end{aligned}
$$

The reflection coefficients are given as $r_{1}=D_{\mathrm{h}}^{(1)} / D_{0}^{(1)}$ and $r_{2}=D_{\mathrm{h}}^{(2)} / D_{0}^{(2)}$, with $D_{0}^{(i)}$ and $D_{\mathrm{h}}^{(i)}$ being the amplitudes of electric displacement of the $i$ th branch of the transmitted and diffracted waves, respectively. $W_{\mathrm{s} 1}$ and $W_{\mathrm{s} 2}$ are the deviation parameters for the beams $\mathbf{S}_{1}{ }^{\prime}$ and $\mathbf{S}_{1}{ }^{\prime \prime}$ at the entrance surface, and $W_{\mathrm{b} 1}$ and $W_{\mathrm{b} 2}$ are those at the back surface. The intensity of IFRB from $\mathrm{A}_{2}$ in Fig. 2(b) is given by

$$
P_{\mathrm{r}}=\left|E_{\mathrm{BbB}}+E_{\mathrm{B} 3 \mathrm{bB}}\right|^{2}=\left[R_{1}^{2}+R_{2}^{2}+2 R_{1} R_{2} \cos (2 \pi \Delta \theta)\right]\left|E_{0}\right|^{2} .
$$

The phase factor $\Delta \theta$ is given by

$$
\Delta \theta=\left(\theta_{x 2}-\theta_{x 1}\right)+\left(\theta_{z 2}-\theta_{z 1}\right)
$$

\section{Discussion}

4.1. Comparison between measured and calculated interference fringes

Intensities of IFRB calculated using equation (16) are shown in Figs. $6(a), 6(b), 6(c)$ for $\beta=-0.02,-0.01$ and $0.0 \mathrm{~mm}^{-1}$, respectively. The integer value at each peak denotes the value of $\Delta \theta$ in equation (17). The distance between two adjacent peaks increases as the distance $x$ increases or the value of $|\beta|$ decreases. The inset of Fig. 6 shows the variations of IFRB (the solid curve) for $\beta=-0.02 \mathrm{~mm}^{-1}$ in the range of $x$ between 5.5 and $8.5 \mathrm{~mm}$, which corresponds to the shaded area in Fig. 6(a). For comparison, the calculated intensity variation of IFMRB is also shown for $\beta=0.02 \mathrm{~mm}^{-1}$. The IFMRB intensities are calculated by assuming the interference between the mirage diffraction beam $\mathbf{S}_{\text {m1 }}^{\prime}$ and the beam $\mathbf{S}_{2}^{\prime}$ reflected from the back surface in Fig. 1(b) and using the formula given by Jongsukswat et al. (2012b). The number of interference fringes of IFRB is ten and that of IFMRB is three. The distance between two adjacent peaks increases as the distance $x$ increases in both the IFRB and IFMRB. The calculated results in Fig. 6(a) and the inset agree quite well with the observed results in Fig. 4 at least qualitatively. Based on this good agreement the strain gradient parameter of the present crystal is determined to be $\beta=-0.02 \mathrm{~mm}^{-1}$. In order to determine the value of $\beta$ only from IFRB, it is necessary to measure the interference fringes for $x$ more than $17 \mathrm{~mm}$ as the difference in the period of the fringes becomes conspicuous for large $x$ as can be seen in Fig. 6. It is possible for the beams

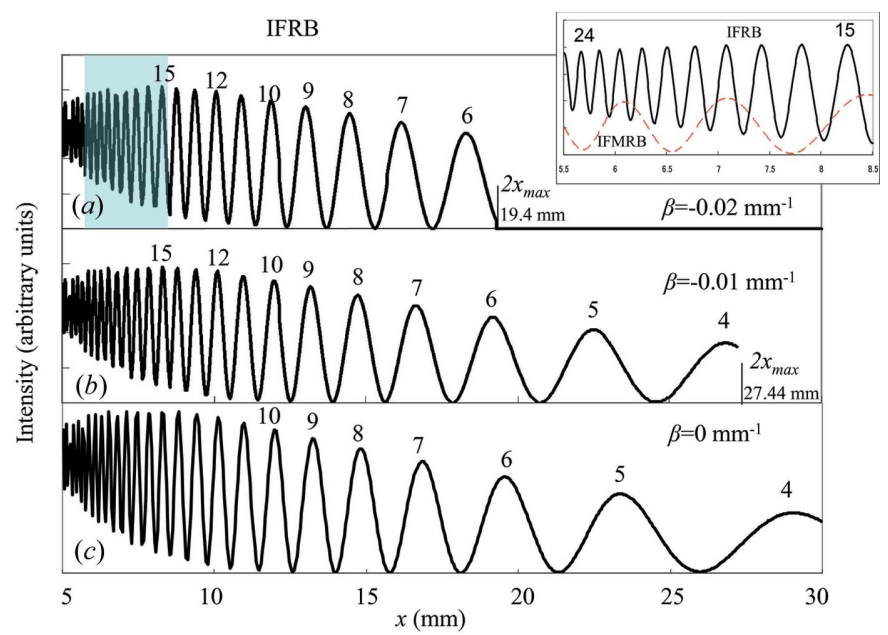

Figure 6

Calculated intensities of IFRB in the range of $x$ from 5 to $30 \mathrm{~mm}$ for $\beta$ being $(a)-0.02 \mathrm{~mm}^{-1},(b)-0.01 \mathrm{~mm}^{-1}$ and $(c) 0.0 \mathrm{~mm}^{-1}$. The inset shows the calculated intensities of IFRB (black curve) for $\beta=$ $-0.02 \mathrm{~mm}^{-1}$ and IFMRB for $\beta=0.02 \mathrm{~mm}^{-1}$ (red dashed curve) in the range of $x$ from 5.5 to $8.5 \mathrm{~mm}$ [the shaded region in $(a)$ ]. 
$\mathbf{S}_{\mathrm{m} 1}^{\prime}$ and $\mathbf{S}_{2}^{\prime}$ (in the BbB mode) to interfere with the beam in the B3bB mode to form the IFMRB. However, as described in Section 3.2, the amplitude of the beam in the B3bB mode is much smaller than that of the mirage diffraction beam $\mathbf{S}_{\mathrm{m} 1}^{\prime}$ because of three more reflections than $\mathbf{S}_{\mathrm{m} 1}^{\prime}$.

The calculated intensities of IFTB are shown in Figs. 7(a), 7(b), 7(c) for $\beta=-0.02,-0.01$ and $0 \mathrm{~mm}^{-1}$, respectively. The distance between two adjacent peaks increases as $x$ increases or $|\beta|$ decreases. There are five peaks in the shaded range of $x$ between 5.5 and $8.5 \mathrm{~mm}$ in Fig. 7(a), which agrees with the experimental result that there are five dark lines in Fig. 5(a) in the same range. The distance between two adjacent peaks in IFTB for $\beta<0$ in Fig. 5(a) is nearly the same as that for $\beta>0$ in Fig. $5(b)$ when $|\beta|$ is the same. Beam trajectories are schematically shown for $\beta<0$ (the black solid curve) and for $\beta>0$ (the red dashed curve) for the same $|\beta|$ in Fig. 8. The relations $W_{\mathrm{s}}(\beta<0)=W_{\mathrm{b}}(\beta>0)$ and $W_{\mathrm{s}}(\beta>0)=W_{\mathrm{b}}(\beta<0)$ hold and the path lengths of the two trajectories [from $\mathrm{A}$ to $\mathrm{A}^{\prime}$ for $\beta<0$ (black solid curve) and $\mathrm{A}^{\prime}$ to $\mathrm{A}$ for $\beta>0$ (red dashed curve)] are the same. The values of $\Delta \theta$ are the same for these two trajectories. The number of oscillations of IFTB for $\beta<0$ should be the same as that for $\beta>0$ when $|\beta|$ is the same.

In Fig. 4 IFLSD are also shown both for $\beta<0(a)$ and $\beta>0$ (b). IFLSD for $\beta<0$ are more clearly observed for small $z$ than for large $z$ in the upper part of Fig. 4(a). The spacing of the two adjacent dark lines is approximately $35 \mu \mathrm{m}$. When $\beta>0$, four dark bands are observed between $z=0$ and $H$ as shown in the upper part of Fig. 4(b). The spacing of the two adjacent bands is approximately $70 \mu \mathrm{m}$, which is twice larger than that in Fig. 4(a). The value of $\beta$ in Fig. 4(a) is $-0.02 \mathrm{~mm}^{-1}$

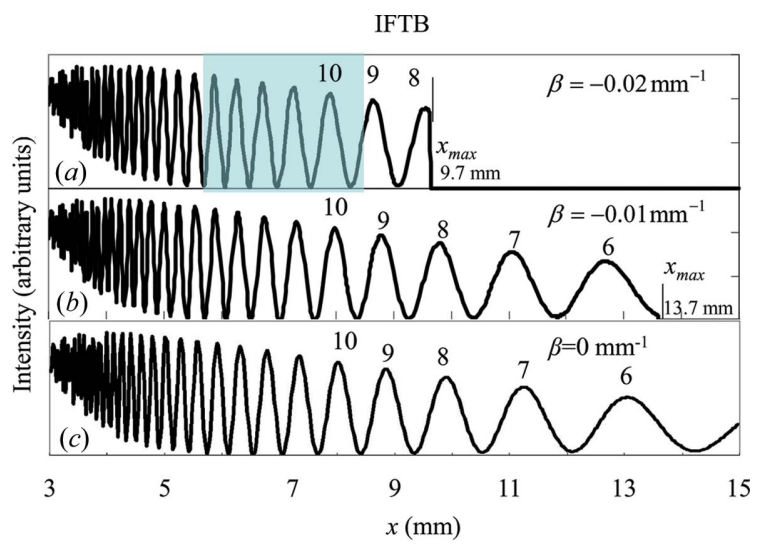

Figure 7

Calculated intensities of IFTB in the range of $x$ from $3 \mathrm{~mm}$ to $x_{\max }$ for $\beta$ being $(a)-0.02 \mathrm{~mm}^{-1},(b)-0.01 \mathrm{~mm}^{-1}$ and $(c) 0.0 \mathrm{~mm}^{-1}$.

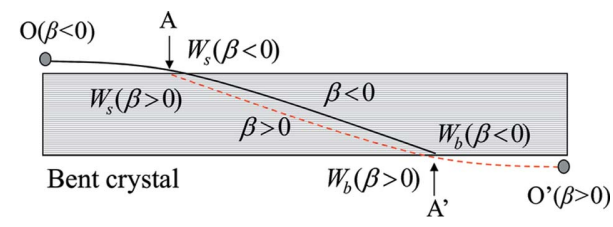

Figure 8

Beam trajectories in a bent plane-parallel crystal for the same $|\beta|$. The black solid curve represents the beam trajectory for $\beta<0$ and the red dashed curve that for $\beta>0$. O is the vertex of the hyperbola for $\beta<0$ and $\mathrm{O}^{\prime}$ that for $\beta>0$. and the corresponding beam trajectories are depicted in Fig. $9(a)$. The beam $\mathbf{S}_{0}$ (the broken curve) corresponding to $W_{\mathrm{s}}=-1$ reaches the lateral surface directly at $z=z_{0}(\simeq$ $0.21 \mathrm{~mm}$ ). No beam in the BL mode comes to the region between $z=0$ and $z_{0}$ on the lateral surface. The notation $\mathrm{L}$ of BL mode denotes that the beam emitted from the lateral surface satisfies the boundary condition of the Laue mode. The observed IFLSD should be formed by interference between the beam $\mathbf{S}_{1}$ in the BbL mode (black solid curve) and the beam $\mathbf{S}_{2}$ in the B2bL mode (red solid curve). The beam trajectories for $\beta=0.02 \mathrm{~mm}^{-1}$ are depicted in Fig. $9(b)$. The beam $\mathbf{S}_{0}$ (thin solid curve) reaches the point $\left(x_{L}, 0\right)$ and the beam $\mathbf{S}_{H}$ (the broken curve) reaches the point $\left(x_{L}, H\right)$. Here, $x_{L}$ is the distance from the incident point to the edge of the crystal surface. If the parameters $W_{\mathrm{s}}$ for $\mathbf{S}_{0}$ and $\mathbf{S}_{H}$ are denoted as $W_{0}$ and $W_{H}$, respectively, the beam $\mathbf{S}_{1}$ is in the BL mode when the parameter $W_{1}\left(W_{\mathrm{s}}\right.$ for $\left.\mathbf{S}_{1}\right)$ satisfies the condition $W_{0}>W_{1}>W_{H}$, and the beam $\mathbf{S}_{2}$ is in the BbL mode when the parameter $W_{2}\left(W_{\mathrm{s}}\right.$ for $\left.\mathbf{S}_{2}\right)$ satisfies $W_{2}<W_{H}$. The IFLSD in Fig. 4(b) is attributed to the interference between the beams in the BL mode (black solid curve) and in the BbL mode (red solid curve).

When $x_{L}$ is larger than the crystal thickness $(H)$, the spacing of two adjacent peaks of IFLSD between the beams in the BL and the BbL modes $\Lambda_{\mathrm{BbL}}$ is given by

$$
\Lambda_{\mathrm{BbL}}=\frac{x_{L} \tan ^{2} \theta_{\mathrm{B}}}{G H},
$$

for $\beta=0$. Here $G$ is the Bragg gap given by $G=$ $\left|\mathbf{K}_{0}\right|\left|\chi_{h}\right| / \cos \theta_{\mathrm{B}}$ with $\left|\mathbf{K}_{0}\right|$ being the wavenumber of the X-ray. Similarly, the spacing of IFLSD between the beams in the BbL and the B2bL modes $\Lambda_{\mathrm{B} 2 \mathrm{bL}}$ is given by

$$
\Lambda_{\mathrm{B} 2 \mathrm{bL}}=\frac{\Lambda_{\mathrm{BbL}}}{2} \text {. }
$$

By using equations (18) and (19), $\Lambda_{\mathrm{B} 2 \mathrm{bL}}$ is calculated to be $31 \mu \mathrm{m}$, which agrees quite well with the measured value of $35 \mu \mathrm{m}$. The crystal was actually bent and $\beta \neq 0$ in the present experiment. But the obtained value of $31 \mu \mathrm{m}$ cannot be much different from the actual value, because equation (19) is a good approximation when $|\beta|$ is very small and the trajectories $\mathbf{S}_{1}$ and $\mathbf{S}_{2}$ are almost straight.

(a)

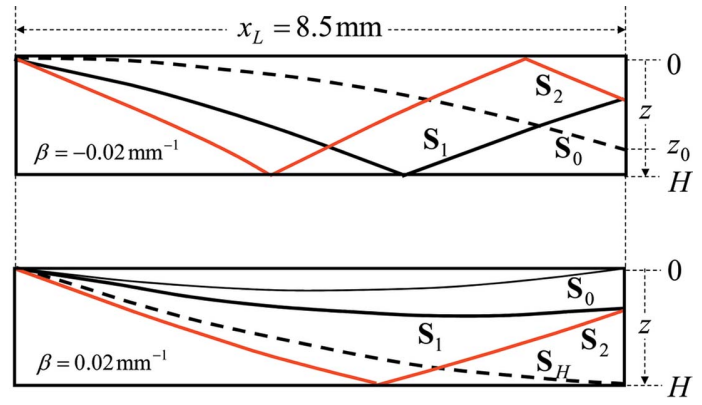

Figure 9

The calculated trajectories of the beams to form IFLSD and IFLST for $(a) \beta=-0.02 \mathrm{~mm}^{-1}$ and $(b) \beta=0.02 \mathrm{~mm}^{-1} . x_{L}=8.5 \mathrm{~mm}$ and $H=$ $0.28 \mathrm{~mm}$. 
By using equations (4) and (5), the values of strain gradient and strain on the crystal surface $(z=0)$ are estimated to be $\varepsilon_{z z}^{\prime}$ $=1.9 \times 10^{-6} \mathrm{~mm}^{-1}$ and $\varepsilon_{z z}=-2.7 \times 10^{-7}$, when the value of $|\beta|$ is $0.02 \mathrm{~mm}^{-1}$. This value is consistent with the previous result by Jongsukswat et al. (2012b). If we assume that the strain is only due to gravity, the value of $|\beta|$ at the distance $l$ between 3.25 and $4 \mathrm{~mm}$ is $9.35 \times 10^{-4} \mathrm{~mm}^{-1}$ using the Young's modulus (105-185 GPa) and Poisson's ratio (0.17-0.33) of Si. This is approximately $5 \%$ of the measured value. On the other hand, the value of $|\beta|$ due to the residual strain by Jongsukswat et al. (2013) is $82.3 \times 10^{-4} \mathrm{~mm}^{-1}$, which is approximately half but close to the current measured value. By considering that residual strain varies depending on the shape, processing, use history and so on, the measured strain should be attributed to mostly residual strain and partly (5\%) to strain due to gravity.

\subsection{Angular amplification of IFRB for $\beta<0$}

The angular amplification rate $\Delta \gamma / \Delta \alpha$, which is defined as the ratio of the divergence angle of the refracted beam $\Delta \gamma$ to that of the incident wave $\Delta \alpha$, has been analytically derived by Authier (2001) in the Laue geometry for a non-absorbing crystal. The rate is much larger at the centre $(W \simeq 0)$ of the reflection region than at the edges $(|W| \simeq 1)$. In the symmetric Bragg geometry, the relation between $W$ and $\gamma$ is given by

$$
\tan \gamma=\frac{\left(W^{2}-1\right)^{1 / 2}}{|W|} \tan \theta_{\mathrm{B}},
$$

where $\gamma$ is the angle of the refracted-beam (the Poynting vector) direction from the direction parallel to the surface. The value of $\gamma$ is 0 for $|W|=1$ and increases as $|W|$ increases. It is close to $\theta_{\mathrm{B}}$ for $|W| \gg 1$. The angular amplification in the symmetric Bragg geometry $A_{\mathrm{B}}$ can be written as

$$
A_{\mathrm{B}}=\left|\frac{\Delta \gamma}{\Delta \alpha}\right|=A_{\mathrm{L}}^{\max } \frac{\cos ^{2} \theta_{\mathrm{B}}}{\left(W^{2}-\sin ^{2} \theta_{\mathrm{B}}\right)\left(W^{2}-1\right)^{1 / 2}},
$$

for $|W|>1$. Here $A_{\mathrm{L}}^{\max }$ is the maximum angular amplification in the symmetric Laue geometry given by

$$
A_{\mathrm{L}}^{\max }=\frac{2 \sin ^{2} \theta_{\mathrm{B}}}{\left|\chi_{h}\right|} .
$$

For $\mathrm{Si}(220)$, the value of $A_{\mathrm{L}}^{\max }$ is $3.5 \times 10^{4}$. In the Bragg geometry, $A_{\mathrm{B}}$ increases as $\Delta W$ approaches zero, and it is

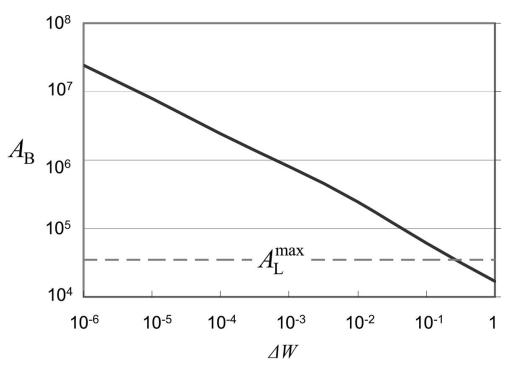

Figure 10

The angular amplification rate $\left(A_{\mathrm{B}}\right)$ as a function of $\Delta W$ for the $\operatorname{Si}(220)$ reflection in the Bragg mode. The solid line shows the value of $A_{\mathrm{B}}$ in equation (21) and the dashed line shows the value of $A_{\mathrm{L}}^{\max }$. infinite at $\Delta W=0$, where $\Delta W$ is defined by $W=-(1+\Delta W)$. Fig. 10 shows values of $A_{\mathrm{B}}$ as a function of $\Delta W$. When $\Delta W$ is $10^{-3}, A_{\mathrm{B}}$ is $7.8 \times 10^{5}$ and 22 times larger than $A_{\mathrm{L}}^{\max }$. In IFMD for $\beta>0$, the typical value of $\Delta W$ corresponding to the first peak $(\Delta \theta=0.5)$ is of the order of $10^{-1}$. On the other hand, in IFRB for $\beta<0, \Delta W$ corresponding to the sixth peak $(\Delta \theta=6)$ in Fig. $6(a)$ is of the order of $10^{-5}$. In order to obtain a high angular amplification rate, it is better to use the interference fringes for $\beta<0$.

\subsection{Coherent condition and energy width of IFRB}

In the present optical system in Fig. 3(a), the divergent angle of the X-rays is estimated to be $670 \mathrm{nrad}$, as the distance from the source to the slit is $30 \mathrm{~m}$ and that from the slit to the sample is $300 \mathrm{~mm}$. The X-ray enters the sample crystal after being reflected from the double-crystal monochromator and passing through the slit. The source size of the X-ray is $60 \mu \mathrm{m}$ and larger than the width of slit $1(20 \mu \mathrm{m})$. In order to discuss the coherence in this case, the effective coherence length and the source size are evaluated using the optical system in Fig. 11. At the incident point $\left(\mathrm{A}_{0}\right)$ of the X-ray, the beams in the $\mathrm{BbB}$ (solid line) and $\mathrm{B} 3 \mathrm{bB}$ modes enter the crystal with the glancing angles $\alpha_{E}(\mathrm{BbB})$ and $\alpha_{E}(\mathrm{~B} 3 \mathrm{bB})$. The angle $\alpha_{E}$ is the glancing angle of incidence for the $\mathrm{X}$-ray with an energy spread of $\delta E$ and $\alpha$ is the glancing angle for the monochromatic X-ray. In other words, $\alpha_{E}$ is the angle in the energydispersive mode and $\alpha$ is that in the rotating-crystal mode. In the experiment, the incident glancing angle $(\alpha)$ is fixed and the $\mathrm{X}$-ray from the bending magnet is a wave having an energy spread of $\delta E$ and the corresponding deviation of wavenumber $\left|\delta \mathbf{K}_{E}\right|$. The divergent angle of the X-ray at the incident point on the crystal surface is related to the energy width $\delta E$, as discussed by Fukamachi et al. $(2014,2015)$. The deviations $\delta E$ and $\left|\delta \mathbf{K}_{E}\right|$ are related to the deviation of the Bragg angle $\delta \theta_{\mathrm{B}}$ in the crystal as

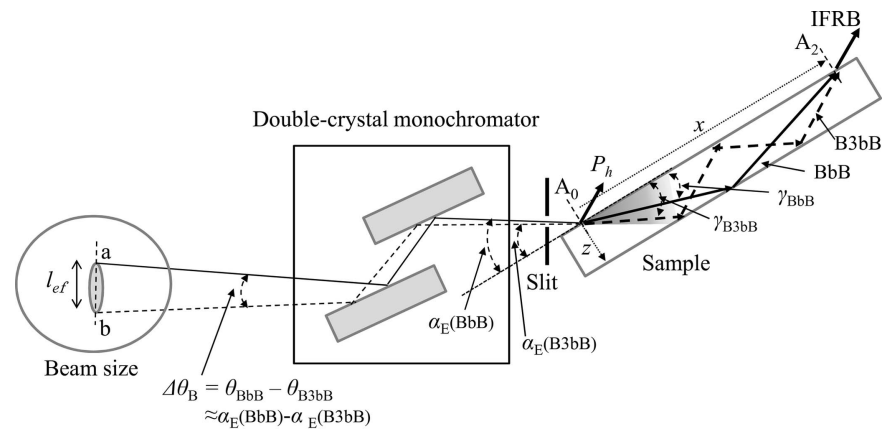

Figure 11

The optical system for evaluating the effective source size and the effective divergent angle for forming IFRB between the beams in the $\mathrm{BbB}$ and $\mathrm{B} 3 \mathrm{bB}$ modes. By tracing the beam in the $\mathrm{BbB}$ mode incident on the sample at $x$ with the glancing angle $\theta_{\mathrm{BbB}}$ back to the source, it arrives at point $a$ in the plane of the source. In a similar way the beam in the $\mathrm{B} 3 \mathrm{bB}$ modes arrives at point $b$. The length between the points $a$ and $b$ gives the effective source size for forming the IFRB at $x$. The effective divergent angle $\Delta \theta_{\mathrm{B}}$ is given by $\theta_{\mathrm{BbB}}-\theta_{\mathrm{B} 3 \mathrm{bB}}$. 


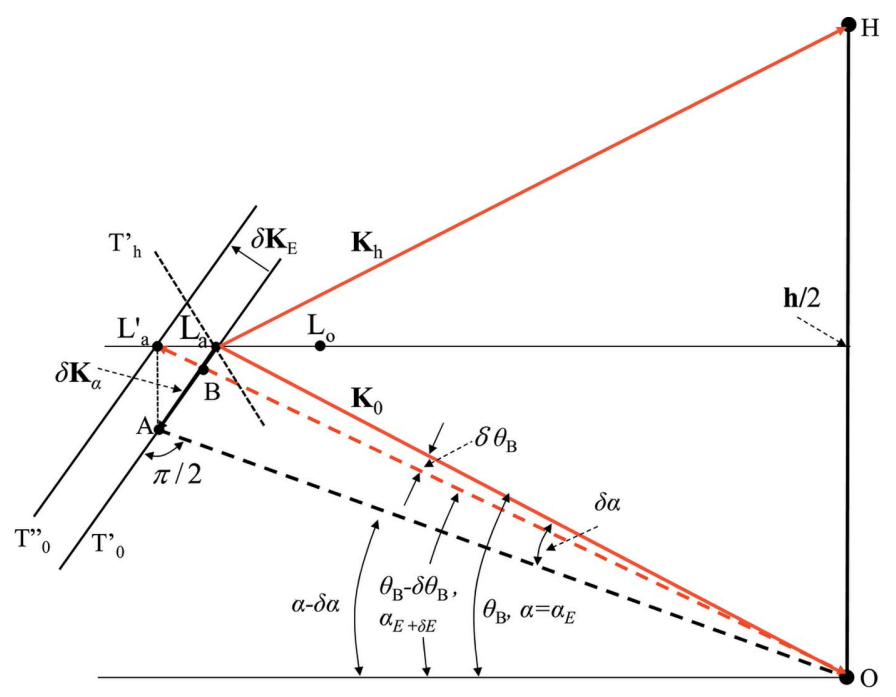

Figure 12

Dispersion surfaces and diffraction geometry in the vacuum. The glancing angle $\alpha$ is taken to be the same as the Bragg angle $\theta_{\mathrm{B}}$, for simplicity. $\mathrm{T}_{0}^{\prime}$ and $\mathrm{T}^{\prime \prime}{ }_{0}$ represent the dispersion surface for the beam with $\mathbf{K}_{0}$ and that with $\mathbf{K}_{0}+\delta \mathbf{K}_{E}$, respectively. $T_{\mathrm{h}}^{\prime}$ represents the dispersion surface for the beam $\mathbf{K}_{\mathrm{h}} . \mathrm{LaO}, \mathrm{L}^{\prime} \mathrm{aO}$ and $\mathrm{AO}$ represent the vectors $\mathbf{K}_{0}$, $\mathbf{K}_{0}+\delta\left(\mathbf{K}_{E}\right)_{/ /} / \cos \theta_{\mathrm{B}}$ and $\mathbf{K}_{0}+\delta \mathbf{K}_{\alpha}$, where $\delta\left(\mathbf{K}_{E}\right)_{/ /}$is the component vector of $\delta \mathbf{K}_{E}$ parallel to the lattice plane.

$$
\frac{|\delta E|}{E}=\left|\frac{\delta \mathbf{K}_{E}}{\mathbf{K}_{0}}\right|=\frac{\delta \theta_{\mathrm{B}}}{\tan \theta_{\mathrm{B}}} .
$$

The divergent angle of the incident $\mathrm{X}$-ray corresponds to the deviation of the Bragg angle $\delta \theta_{\mathrm{B}}$. For relating $\delta \theta_{\mathrm{B}}$ to $\delta \alpha$, Fig. 12 shows the dispersion surfaces for the incident wavevectors $\mathbf{K}_{0}$ and $\mathbf{K}_{0}+\delta \mathbf{K}_{E}$ with the glancing angle $\alpha$ and the diffraction geometry. For simplicity, the glancing angle $\alpha$ is assumed to be the same as $\theta_{\mathrm{B}}$. The dispersion surfaces and the diffraction geometry in the more general case are given by Fukamachi et al. $(2014,2015)$. The vector $\overrightarrow{\mathrm{OH}}$ is the reciprocal-lattice vector $\mathbf{h}$ and $\mathbf{K}_{\mathrm{h}}$ the wavevector of the diffracted wave. The lines $\mathrm{T}_{0}^{\prime}$ and $\mathrm{T}^{\prime \prime}{ }_{0}$ represent the dispersion surfaces for the wavevectors $\mathbf{K}_{0}$ and $\mathbf{K}_{0}+\delta \mathbf{K}_{E}$, respectively. $\mathrm{L}_{a}$ and $\mathrm{L}^{\prime}{ }_{a}$ are the corresponding Laue points and the distance $\mathrm{L}_{a} \mathrm{~L}^{\prime}{ }_{a}$ is given by $\left|\delta \mathbf{K}_{E}\right| / \cos \theta_{\mathrm{B}}$. To obtain the same variation of $\mathrm{L}_{a} \mathrm{~L}^{\prime}{ }_{a}$ by changing the incident angle $\alpha(\delta \alpha)$, the component of the wavevector $\delta \mathbf{K}_{\alpha}$ parallel to the lattice plane $\left(\left|\delta \mathbf{K}_{\alpha}\right| \sin \theta_{\mathrm{B}}\right)$ must be $\mathrm{L}_{a} \mathrm{~L}^{\prime}{ }_{a}$. Then the relations $\left|\delta \mathbf{K}_{\alpha}\right| \sin \theta_{\mathrm{B}}=\left|\mathbf{K}_{0}\right| \delta \alpha \sin \theta_{\mathrm{B}}=\left|\delta \mathbf{K}_{E}\right| / \cos \theta_{\mathrm{B}}$ hold. Using these relations and equation (23), $\delta \theta_{\mathrm{B}}$ is related to $\delta \alpha$ as

$$
\left|\delta \theta_{\mathrm{B}}\right|=\sin ^{2} \theta_{\mathrm{B}}|\delta \alpha|
$$

The energy deviation is related to $\delta W$ as

$$
\frac{|\delta E|}{E}=\frac{\left|\chi_{h}\right|}{2}|\delta W|
$$

by using the relation

$$
\delta W=\frac{\sin 2 \theta_{\mathrm{B}}}{\left|\chi_{h}\right|} \delta \alpha
$$

obtained from equation (1) by setting $C=1$. The effective angle divergence $\delta \theta_{\mathrm{B}}$ of the incident beam is related to $\delta W$ as
Table 1

The deviation parameters $\Delta W_{\mathrm{BbB}}$ and $\Delta W_{\mathrm{B} 3 \mathrm{bB}}$ for the beams in the $\mathrm{BbB}$ and the B3bB modes at position $x$ from 5 to $9 \mathrm{~mm}$ in Fig. 4(a) (in the second and the third columns, respectively).

The corresponding effective divergent angle $\Delta \theta_{\mathrm{B}}$, source size $l_{\mathrm{ef}}$, energy width $\delta E$ and longitudinal coherence length $l_{\mathrm{L}}$ for formation of the IFRB between these two beams are in the fourth to the seventh columns. $\Delta l_{\mathrm{p}}$ in the last column is the path length difference between these two beams.

\begin{tabular}{llllllll}
\hline $\begin{array}{l}x \\
(\mathrm{~mm})\end{array}$ & $\Delta W_{\mathrm{BbB}}$ & $\Delta W_{\mathrm{B} 3 \mathrm{bB}}$ & $\begin{array}{l}\Delta \theta_{\mathrm{B}} \\
(\mathrm{nrad})\end{array}$ & $\begin{array}{l}l_{\mathrm{ef}} \\
(\mu \mathrm{m})\end{array}$ & $\begin{array}{l}\delta E \\
(\mathrm{meV})\end{array}$ & $\begin{array}{l}l_{\mathrm{L}} \\
(\mu \mathrm{m})\end{array}$ & $\begin{array}{l}\Delta l_{\mathrm{p}} \\
(\mu \mathrm{m})\end{array}$ \\
\hline 5 & 0.07 & 0.47 & 300 & 9 & 10.8 & 51 & 93 \\
6 & 0.04 & 0.26 & 166 & 5 & 6 & 92 & 78 \\
7 & 0.03 & 0.17 & 105 & 3.1 & 3.8 & 146 & 67 \\
8 & 0.02 & 0.12 & 75 & 2.3 & 2.7 & 204 & 58 \\
9 & 0.01 & 0.09 & 60 & 1.8 & 2.2 & 255 & 52 \\
\hline
\end{tabular}

$$
\delta W=\frac{2}{\left|\chi_{h}\right| \tan \theta_{\mathrm{B}}} \delta \theta_{\mathrm{B}} .
$$

The divergent angle of the beam from a point source corresponds to the deviation of $\delta \alpha_{E}$ and the deviation of the Bragg angle $\delta \theta_{\mathrm{B}}$ in the present experiment, which gives rise to the deviation of $|\delta W|=0.90$ for the $\mathrm{Si}(220)$ reflection.

In order to compare the range of $|\delta W|$ with that needed to excite the refracted beams in the $\mathrm{BbB}$ and $\mathrm{B} 3 \mathrm{bB}$ modes coherently, the values of $\Delta W_{\mathrm{BbB}}$ and $\Delta W_{\mathrm{B} 3 \mathrm{bB}}$ for the beams in the $\mathrm{BbB}$ mode and the $\mathrm{B} 3 \mathrm{bB}$ mode are determined by using equation (2) and $\beta=-0.02 \mathrm{~mm}^{-1}$. Here $\Delta W_{\mathrm{BbB}}$ and $\Delta W_{\mathrm{B} 3 \mathrm{bB}}$ are $\Delta W$ for the beams in $\mathrm{BbB}$ mode and $\mathrm{B} 3 \mathrm{bB}$ mode, respectively. The values of $\Delta W_{\mathrm{BbB}}$ and $\Delta W_{\mathrm{B} 3 \mathrm{bB}}$ for $x$ from 5 to $9 \mathrm{~mm}$ are listed in the second and third columns, respectively, in Table 1. The range of $|\delta W|=\left|\Delta W_{\mathrm{BbB}}-\Delta W_{\mathrm{B} 3 \mathrm{bB}}\right|$ for exciting the IFRB in the range of $x$ from 5 to $9 \mathrm{~mm}$ is 0.46 and smaller than $|\delta W|=0.90$. The incident $\mathrm{X}$-ray with the divergent angle of $670 \mathrm{nrad}$ is enough to excite the relevant refracted beams in the $\mathrm{BbB}$ and $\mathrm{B} 3 \mathrm{bB}$ modes coherently.

Next, the longitudinal coherent condition is discussed. In the present experiment, the values of $\gamma$ and the path lengths are different for the beams in the $\mathrm{BbB}$ and $\mathrm{B} 3 \mathrm{bB}$ modes as shown in Fig. $2(b)$. The value of $\gamma_{\mathrm{BbB}}\left(\gamma_{\mathrm{B} 3 \mathrm{bB}}\right)$ is obtained by putting the value of $W_{\mathrm{BbB}}\left(W_{\mathrm{B} 3 \mathrm{bB}}\right)$ into equation (20). The path length of the beam in the $\mathrm{BbB}(\mathrm{B} 3 \mathrm{bB})$ mode is calculated using the values of $\gamma_{\mathrm{BbB}}\left(\gamma_{\mathrm{B} 3 \mathrm{bB}}\right)$ by assuming that the beam trajectory is straight, because the hyperbolic curvature is small. The path length differences $\Delta l_{\mathrm{p}}$ thus calculated from the path lengths of these two beams are listed in the last column of Table 1. As the longitudinal coherence length $l_{\mathrm{L}}$ (Born \& Wolf, 1970) is given by

$$
l_{\mathrm{L}}=\frac{\lambda}{2}\left(\frac{E}{\delta E}\right),
$$

the value is obtained by inserting equation (25) into equation (27) and using $|\delta W|$. The longitudinal coherence length $l_{\mathrm{L}}$ for these beams is listed in the seventh column of Table 1. At $x=$ $8 \mathrm{~mm}$, the path length difference is $58 \mu \mathrm{m}$ and the coherence length is $204 \mu \mathrm{m}$. The coherence length is larger than the path length difference, and the coherence condition is satisfied. On 
the other hand, the coherence condition is not fully satisfied at $x=5 \mathrm{~mm}$, as the coherence length is $51 \mu \mathrm{m}$ and the path length difference is $93 \mu \mathrm{m}$. The interference fringe is blurred around $x=5 \mathrm{~mm}$ in Fig. 4(a).

The transverse coherence length $l_{\mathrm{T}}$ is given by

$$
l_{\mathrm{T}}=\frac{\lambda}{2 \Delta \theta_{\mathrm{B}}},
$$

where $\Delta \theta_{\mathrm{B}}=\theta_{\mathrm{B} 3 \mathrm{bB}}-\theta_{\mathrm{BbB}}$ with $\theta_{\mathrm{BbB}}$ and $\theta_{\mathrm{B} 3 \mathrm{bB}}$ being the Bragg angles for the beams in the $\mathrm{BbB}$ and $\mathrm{B} 3 \mathrm{bB}$ modes, respectively. The transverse coherence length $l_{\mathrm{T}}$ in the present experiment is $186 \mu \mathrm{m}$. The effective source size $\left(l_{\mathrm{ef}}\right)$ is given by the product of $\Delta \theta_{\mathrm{B}}$ with the distance from the source point to the sample (approximately $30 \mathrm{~m}$ ). The calculated values of $\Delta \theta_{\mathrm{B}}$ and $l_{\mathrm{ef}}$ are listed in the fourth and fifth columns, respectively, of Table 1 . As the maximum effective source size is $l_{\text {ef }}=$ $9 \mu \mathrm{m}$ for the formation of IFRB at $x=5 \mathrm{~mm}$, the coherence condition is satisfied.

In the observation of IFRB, there is another possible blurring effect caused by the different travelling directions between the beams in the $\mathrm{BbB}$ and the $\mathrm{B} 3 \mathrm{bB}$ modes. The difference in the directions of these two beams is approximately $300 \mathrm{nrad}$. As the distance from the sample to the nuclear plate is $100 \mathrm{~mm}$, the resultant difference in the arriving positions on the plate is $30 \mathrm{~nm}$. This difference is much smaller than a period of the IFTB of $0.3 \mathrm{~mm}$ and the blurring effect is negligible. A similar blurring effect is expected in the observation of IFMRB and IFTB. This is also negligible in the present setup.

\section{Summary}

The trajectory of the X-ray refracted beam in a very weakly bent perfect crystal with negative strain gradient shows a hyperbolic form opening down, while it shows a hyperbolic form opening up when the strain gradient is positive. This difference in beam trajectory results in quite different X-ray interference fringes. The interference fringes for $\beta<0$ were mainly studied in the above. In the wave emitted from the entrance surface IFRB were observed between once- and twice-reflected beams from the back surface. In the transmitted wave from the back surface, IFTB were observed between the beam directly reaching the back surface and the beam once reflected from the entrance surface. These interference fringes for $\beta<0$ were compared with the corresponding interference fringes for $\beta>0$. IFRB and IFTB were analysed by using the dynamical theory of diffraction for a bent crystal. The results showed very good agreement between the observed and the calculated values of the interference fringe spacing, which enabled us to evaluate the strain gradient of the sample crystal. If a thinner crystal is used when $\beta \leq 0$, it is possible to observe Bragg-Pendellösung fringes reported by Batterman \& Hildebrandt (1968), which are formed by interference between two waves corresponding to the two branches of the dispersion surface as shown by Authier (2008). But if $\beta>0$, it is not possible to observe them because of the mirage effect.

The fringe spacing of IFRB for $\beta<0$ is smaller than that of IFMRB for $\beta>0$. It is noted that IFRB for $\beta<0$ should be potentially useful for measuring a very weak strain of the order of $10^{-7}$.

\section{Funding information}

This work was carried out under the approval of the Program Advisory Committee of PF (Proposal No. 2012G606). This work was partially supported by the Nano Technology Project for Private Universities (2011-2013): matching fund subsidy from the Ministry of Education, Culture, Sports, Science and Technology.

\section{References}

Authier, A. (2001). Dynamical Theory of X-ray Diffraction. Oxford University Press.

Authier, A. (2008). Acta Cryst. A64, 337-340.

Batterman, B. W. \& Hildebrandt, G. (1968). Acta Cryst. A24, 150-157.

Born, M. \& Wolf, E. (1970). Principles of Optics. Oxford: Pergamon Press.

Fukamachi, T., Hirano, K., Negishi, R., Kanematsu, Y., Jongsukswat, S., Hirano, K. \& Kawamura, T. (2011). Acta Cryst. A67, 154-159.

Fukamachi, T., Jongsukswat, S., Ju, D., Negishi, R., Hirano, K. \& Kawamura, T. (2014). J. Appl. Cryst. 47, 1267-1272.

Fukamachi, T., Jongsukswat, S., Ju, D., Negishi, R., Hirano, K. \& Kawamura, T. (2015). J. Appl. Cryst. 48, 312.

Fukamachi, T., Jongsukswat, S., Kanematsu, Y., Hirano, K., Negishi, R., Shimojo, M., Ju, D., Hirano, K. \& Kawamura, T. (2011). J. Phys. Soc. Jpn, 80, 083002.

Fukamachi, T., Negishi, R., Yoshizawa, M. \& Kawamura, T. (2005). Jpn. J. Appl. Phys. 44, L787-L789.

Fukamachi, T., Negishi, R., Yoshizawa, M., Sakamaki, T. \& Kawamura, T. (2004). Jpn. J. Appl. Phys. 43, L865-L867.

Fukamachi, T., Tohyama, M., Hirano, K., Yoshizawa, M., Negishi, R., Ju, D., Hirano, K. \& Kawamura, T. (2010). Acta Cryst. A66, 421426.

Gronkowski, J. \& Malgrange, C. (1984). Acta Cryst. A40, 507-514.

Hirano, K., Fukamachi, T., Yoshizawa, M., Negishi, R., Hirano, K. \& Kawamura, T. (2009a). Acta Cryst. A65, 253-258.

Hirano, K., Fukamachi, T., Yoshizawa, M., Negishi, R., Hirano, K. \& Kawamura, T. (2009b). Phys. Status Solidi A, 206, 1855-1859.

Hirano, K., Fukamachi, T., Yoshizawa, M., Negishi, R., Hirano, K., Xu, Z. \& Kawamura, T. (2008). J. Phys. Soc. Jpn, 77, 103707.

Jongsukswat, S., Fukamachi, T., Hirano, K., Ju, D., Negishi, R., Shimojo, M., Hirano, K. \& Kawamura, T. (2012a). Jpn. J. Appl. Phys. 51, 076702.

Jongsukswat, S., Fukamachi, T., Hirano, K., Ju, D., Negishi, R., Shimojo, M., Hirano, K. \& Kawamura, T. (2012b). J. Phys. Soc. Jpn, 81, 094804.

Jongsukswat, S., Fukamachi, T., Ju, D., Negishi, R., Hirano, K. \& Kawamura, T. (2013). J. Appl. Cryst. 46, 1261-1265.

Yan, H., Kalenci, Ö. \& Noyan, I. C. (2007). J. Appl. Cryst. 40, 322-331. Yan, H. \& Noyan, I. C. (2006). J. Appl. Cryst. 39, 320-325. 